ARTIGO ORIGINAL

CAROLINA MARTINS COSTA

MAIRA OLIVEIRA BITENCOURT

ALEXANDRE FERREIRA BELLO

ALEXANDRE PAIM DIAZ
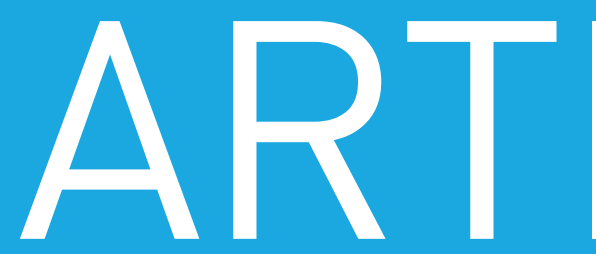

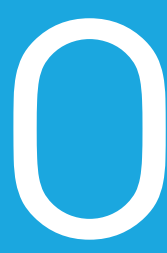

\title{
TAXAS DE MORTALIDADE POR SUICÍDIO, DOENC̣A CEREBROVASCULAR, DOENCAA ISQUÊMICA DO CORACCÃO E DOENCCA CRÔNIĆA DE VIAS AÉREAS SUPERIORES EM IDOSOS BRASILEIROS: UMA ANÁLISE TEMPORAL DE 20 ANOS
}

\section{MORTALITY RATES FROM SUICIDE, CEREBROVASCULAR DISEASE, ISCHEMIC HEART DISEASE, AND CHRONIC UPPER AIRWAY DISEASE IN BRAZILIAN ELDERLY: A 20-YEAR} TEMPORAL ANALYSIS

\section{Resumo}

Objetivo: Investigar a tendência das taxas de suicídio em idosos brasileiros em um período de 20 anos em relação às principais causas de mortalidade nessa população.

Métodos: Nesta análise de tendência temporal, dados sobre o número de óbitos foram obtidos do Sistema de Informações sobre Mortalidade (SIM) do DATASUS. As taxas de mortalidade foram calculadas com dados obtidos do Instituto Brasileiro de Geografia e Estatística (IBGE). Foram calculadas as taxas de mortalidade em idosos por suicídio e para as três principais causas de mortalidade no ano de início do período de investigação (1996), estratificadas por sexo e faixa etária. Para estimar a tendência temporal, foi utilizada regressão linear simples.

Resultados: Encontramos um aumento significativo nas taxas de suicídio para homens e mulheres entre $60 \mathrm{e}$ 69 anos de idade, além de redução significativa nas taxas de mortalidade por doença cerebrovascular, doença isquêmica do coração e doença crônica de vias aéreas superiores na mesma faixa etária.

Conclusão: É possível que políticas públicas para a redução dos fatores de risco e acesso ao tratamento tenham tido um impacto na redução da mortalidade por doença cerebrovascular, doença isquêmica do coração e doença crônica de vias aéreas superiores em idosos. A redução da mortalidade por suicídio provavelmente exigirá um direcionamento semelhante em relação à saúde mental.

Palavras-chave: Suicídio, idosos, mortalidade.

\section{Abstract}

Objective: To investigate trends in suicide rates among Brazilian elderly over a timeframe of 20 years with regard to the main causes of mortality in this population.

Methods: In this temporal trend analysis, data on the number of deaths were collected from the Mortality Information System (Sistema de Informações de Mortalidade - SIM) that integrates the public national healthcare database DATASUS. Mortality rates were calculated with data obtained from the Brazilian Institute of Geography and Statistics (Instituto Brasileiro de Geografia e Estatística - IBGE). Mortality rates in the elderly were calculated for suicide and for the three main causes of mortality in this population in the first year of the observation (1996), stratified by gender and age range. The temporal trend was estimated using linear regression analysis. 


\section{CAROLINA MARTINS COSTA', MAIRA OLIVEIRA BITENCOURT ${ }^{2}$, ALEXANDRE FERREIRA BELLO ${ }^{1}$, ALEXANDRE PAIM DIAZ ${ }^{3}$}

${ }^{1}$ Faculdade de Medicina, Universidade do Sul de Santa Catarina (UNISUL), Palhoça, SC. ${ }^{2}$ Instituto de Psiquiatria de Santa Catarina, São José, SC. ${ }^{3}$ Universidade Federal de Santa Catarina (UFSC), Florianópolis, SC.

Results: A significant increase was found in suicide rates for both male and female elderly between 60 and 69 years of age, as well as a significant reduction in mortality rates due to cerebrovascular disease, ischemic heart disease and chronic upper airway disease in the same age range.

Conclusion: It is possible that public policies targeted at reducing risk factors and improving access to treatment have had an impact on the reduction of mortality for cerebrovascular disease, ischemic heart disease and chronic upper airway disease in the elderly. Reduction of suicide rates will probably demand a similar approach in relation to mental health.

Keywords: Suicide, elderly, mortality.

\section{INTRODUÇÃO}

A proporção dos idosos em relação à população geral vem crescendo, o que tem sido um dos fenômenos mais notórios em todo o mundo, observando-se uma transformação demográfica sem precedentes na história, trazendo consigo repercussões culturais, sociais e políticas. O Brasil acompanha essa transição: a cada ano, 650 mil novos idosos são incorporados à população brasileira. As projeções para o ano de 2020 estimam-se em 32 milhões, o que situará o Brasil na sexta posição mundial em número de idosos ${ }^{1}$.

A análise da mortalidade entre os idosos evidencia um aumento da longevidade, além da mudança do perfil das causas de mortalidade. Ainda que as doenças do aparelho circulatório permaneçam como as principais causas, suas taxas estão em declínio, o que pode estar associado a mudanças nos hábitos alimentares, redução no tabagismo, prática de atividade física, entre outros fatores de risco para doenças cardiovasculares.

As taxas de suicídio têm sido descritas como crescentes na atualidade, inclusive na população idosa, sendo este considerado um grave problema de saúde pública². Segundo o Relatório Global de Suicídio da Organização Mundial da Saúde de 2014, os índices são maiores entre as pessoas acima de 65-70 anos de idade, cerca do dobro em relação à população geral, com predominância no sexo masculino ${ }^{3,4}$. Entre os principais fatores de risco para o suicídio estão os transtornos psiquiátricos, especialmente a depressão. Estima-se que pelo menos 50\% dos suicídios estejam associados a esse transtorno de humor ${ }^{5}$. Além disso, isolamento social ${ }^{6}$, transição para a aposentadoria, perda de pessoas próximas e maior prevalência de doenças neurodegenerativas também são importantes fatores de risco?.

Nos EUA, as taxas de mortalidade por suicídio vêm aumentando nas últimas décadas², tendência contrária às relacionadas às doenças do coração e câncer ${ }^{8}$. No Brasil, um estudo identificou um aumento nas taxas de suicídio em idosos no período de 1996 a 2013 no Estado da Bahia', e outro mais recente, um aumento nas taxas de suicídio em pessoas acima de 60 anos de idade ${ }^{10}$. No entanto, não encontramos estudos que tenham avaliado tendências nacionais das taxas de suicídio em estratos etários específicos na população idosa, além de uma comparação dessas taxas com outras causas de mortalidade.

Com o processo de transição demográfica e consequente envelhecimento da população, tornase necessário um olhar mais atento para o estudo das principais causas de mortalidade em idosos. Comparar a tendência das taxas de suicídio em idosos em relação às taxas de mortalidade por outras doenças é fundamental para investigar se tais tendências apresentam direção semelhante ou oposta e quais estratégias podem ser apreendidas e implementadas para a redução da mortalidade por causas específicas. Nesse contexto, esse estudo visa avaliar a tendência das taxas de suicídio em idosos brasileiros em um período de 20 anos em relação às principais causas de mortalidade nessa população.

\section{Métodos}

Trata-se de estudo ecológico de tendência temporal. Neste estudo, foi utilizada fonte de dados do Departamento de Informática do Sistema Único de Saúde (DATASUS), que disponibiliza informações sobre o estado de saúde da população. Deste, foram retirados o número absoluto de óbitos conforme a causa base, registrados no Sistema de Informações sobre Mortalidade (SIM). As taxas de mortalidade foram calculadas de acordo com dados obtidos do Instituto Brasileiro de Geografia e Estatística (IBGE): para o ano de 1996, foi considerada contagem populacional; para os anos de 1997-1999, 2001-2009 e 2011-2012, foram consideradas estimativas populacionais; e para os anos 2000 e 2010, censos demográficos. Como não foram encontradas estimativas para os anos de 2013 a 2016, 


\section{ARTIGO ORIGINAL}

\section{CAROLINA MARTINS COSTA MAIRA OLIVEIRA BITENCOURT ALEXANDRE FERREIRA BELLO ALEXANDRE PAIM DIAZ}
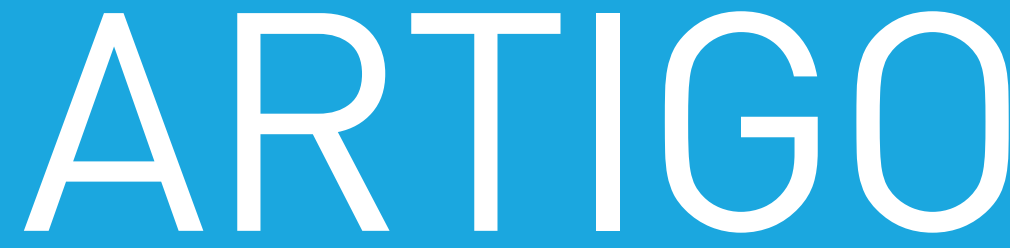

as taxas de mortalidade foram determinadas de acordo com a estimativa da população para o ano de 2012.

Foi selecionado o número de óbitos para três das principais causas de mortalidade em idosos no ano de 1996: doenças isquêmicas do coração (DIC) [10 edição da Classificação Internacional de Doenças (CID-10) I20-125], doenças cerebrovasculares (160-169) e doenças crônicas das vias aéreas inferiores (J40-J47). Além dessas, foi obtido o número de óbitos por lesões autoprovocadas voluntariamente (X60-X84). Os dados são de domínio público e foram totalmente coletados a partir do banco de dados do DATASUS/SIM. As taxas de mortalidade foram calculadas a partir do número de óbitos dividido pela população da respectiva faixa etária, tendo um resultado multiplicado pela constante 100.000. As mortalidades foram estratificadas por sexo e por faixas etárias entre 6064 anos, 65-69 anos, 70-74 anos, 75-79 anos e 80 anos e mais. Tais taxas foram as variáveis dependentes, classificadas como quantitativas contínuas. A variável independente foi o ano. Análise de regressão linear simples foi utilizada para avaliar a tendência das taxas de mortalidade para cada ano. Os dados foram analisados de acordo com o programa Statistical Package for Social Sciences (SPSS) versão 10.0.

O projeto obedece aos preceitos éticos do Conselho Nacional de Saúde (CNS), resolução n 466/2012. Os dados utilizados para a pesquisa são do DATASUS, de domínio público, de acesso irrestrito e sem identificação dos indivíduos, portanto é dispensada a aprovação do Comitê de Ética em Pesquisa envolvendo seres humanos. Não é necessário também o uso do termo de consentimento livre e esclarecido.

\section{Resultados}

A Tabela 1 mostra a variação nas taxas de mortalidade por 100.000 habitantes por sexo, faixa etária e causa de mortalidade entre os anos 1996 e 2016 . Houve uma variação positiva nas taxas de suicídio em homens para todas as faixas etárias analisadas. Para o sexo feminino, houve uma variação positiva na faixa etária de 60 a 69 anos e negativa a partir dos 70 anos de idade (Tabela 1).

Tabela 1 - Variação da taxa de mortalidade por causa e faixa etária específica no período de 1996 a 2016

\begin{tabular}{|c|c|c|c|c|}
\hline \multirow[b]{2}{*}{ Faixa etária } & \multicolumn{4}{|c|}{ Causas de mortalidade (taxa de mortalidade por 100.000 habitantes) } \\
\hline & $\begin{array}{l}\text { Morte por lesão } \\
\text { autoprovocada }\end{array}$ & $\begin{array}{c}\text { Doença } \\
\text { cerebrovascular }\end{array}$ & $\begin{array}{l}\text { Doença isquêmica } \\
\text { do coração }\end{array}$ & $\begin{array}{c}\text { Doença crônica de } \\
\text { vias aéreas inferiores }\end{array}$ \\
\hline \multirow{2}{*}{\multicolumn{5}{|c|}{$\begin{array}{l}\text { Geral (a partir dos } 60 \\
\text { anos de idade) } \\
\text { Masculino }\end{array}$}} \\
\hline & & & & \\
\hline \multicolumn{5}{|c|}{1996} \\
\hline 2016 & 17,53 & 448,84 & 527,03 & 245,95 \\
\hline Variação & $+42,6 \%$ & $-13,35 \%$ & $+4,67 \%$ & $-14,17 \%$ \\
\hline \multicolumn{5}{|l|}{ Feminino } \\
\hline 1996 & 2,77 & 436,19 & 365,59 & 152,63 \\
\hline 2016 & 3,16 & 368,51 & 340,57 & 158,38 \\
\hline Variação & $+14,1 \%$ & $-15,52$ & $-6,84 \%$ & $+3,76 \%$ \\
\hline \multicolumn{5}{|l|}{60 a 64 anos } \\
\hline \multicolumn{5}{|l|}{ Masculino } \\
\hline 1996 & 11,38 & 234,46 & 287 & 97,94 \\
\hline 2016 & 14,67 & 158,84 & 273 & 65,17 \\
\hline Variação & $+28,9 \%$ & $-32,25 \%$ & $-4,88 \%$ & $-33,46 \%$ \\
\hline \multicolumn{5}{|l|}{ Feminino } \\
\hline 1996 & 2,37 & 148,22 & 143,16 & 49,79 \\
\hline 2016 & 3,63 & 97,92 & 117,81 & 44,15 \\
\hline Variação & $+53,2 \%$ & $-33,94 \%$ & $-17,71 \%$ & $-11,33 \%$ \\
\hline
\end{tabular}


${ }^{1}$ Faculdade de Medicina, Universidade do Sul de Santa Catarina (UNISUL), Palhoça, SC. ${ }^{2}$ Instituto de Psiquiatria de Santa Catarina, São José, SC. ${ }^{3}$ Universidade Federal de Santa Catarina (UFSC), Florianópolis, SC.

Tabela 1 - Continua

\begin{tabular}{|c|c|c|c|c|}
\hline \multirow[b]{2}{*}{ Faixa etária } & \multicolumn{4}{|c|}{ Causas de mortalidade (taxa de mortalidade por 100.000 habitantes) } \\
\hline & $\begin{array}{l}\text { Morte por lesão } \\
\text { autoprovocada }\end{array}$ & $\begin{array}{c}\text { Doença } \\
\text { cerebrovascular }\end{array}$ & $\begin{array}{c}\text { Doença isquêmica } \\
\text { do coração }\end{array}$ & $\begin{array}{c}\text { Doença crônica de } \\
\text { vias aéreas inferiores }\end{array}$ \\
\hline \multicolumn{5}{|l|}{65 a 69 anos } \\
\hline \multicolumn{5}{|l|}{ Masculino } \\
\hline 1996 & 11,76 & 360,28 & 403,30 & 184,45 \\
\hline 2016 & 18,79 & 265,13 & 403,86 & 126,10 \\
\hline Variação & $+59,8 \%$ & $-26,41 \%$ & $+0,001 \%$ & $-31,63 \%$ \\
\hline \multicolumn{5}{|l|}{ Feminino } \\
\hline 1996 & 2,71 & 238,33 & 224,89 & 86,00 \\
\hline 2016 & 3,73 & 160,34 & 198,77 & 79,14 \\
\hline Variação & $+37,6 \%$ & $-32,72 \%$ & $-11,61 \%$ & $-7,98 \%$ \\
\hline \multicolumn{5}{|l|}{70 a 74 anos } \\
\hline \multicolumn{5}{|l|}{ Masculino } \\
\hline 1996 & 12,37 & 564,46 & 556,69 & 316,77 \\
\hline 2016 & 17,26 & 408,59 & 510,70 & 214,26 \\
\hline Variação & $+39,53 \%$ & $-27,61 \%$ & $-8,26 \%$ & $-32,36 \%$ \\
\hline \multicolumn{5}{|l|}{ Feminino } \\
\hline 1996 & 3,10 & 396,92 & 346,44 & 144,80 \\
\hline 2016 & 2,76 & 263,76 & 275,98 & 127,48 \\
\hline Variação & $-10,97 \%$ & $-33,55 \%$ & $-20,34 \%$ & $-11,96 \%$ \\
\hline \multicolumn{5}{|l|}{75 a 79 anos } \\
\hline \multicolumn{5}{|l|}{ Masculino } \\
\hline 1996 & 15,17 & 845,38 & 732,70 & 494,50 \\
\hline 2016 & 20,71 & 691,76 & 740,13 & 397,96 \\
\hline Variação & $+36,52$ & $-18,17 \%$ & $+1,01 \%$ & $-19,52 \%$ \\
\hline \multicolumn{5}{|l|}{ Feminino } \\
\hline 1996 & 3,34 & 664,54 & 536,43 & 241,51 \\
\hline 2016 & 2,81 & 484,67 & 445,84 & 221,82 \\
\hline Variação & $-15,87 \%$ & $-27,07 \%$ & $-16,89 \%$ & $-8,15 \%$ \\
\hline \multicolumn{5}{|c|}{ Acima de 80 anos } \\
\hline \multicolumn{5}{|c|}{ Masculino } \\
\hline 1996 & 13,19 & $1.380,40$ & $1.100,43$ & 854,80 \\
\hline 2016 & 39,10 & $1.415,49$ & $1.271,84$ & 867,63 \\
\hline Variação & $+196 \%$ & $+2,54 \%$ & $+15,58 \%$ & $+1,50 \%$ \\
\hline \multicolumn{5}{|l|}{ Feminino } \\
\hline 1996 & 2,90 & $1.380,98$ & $1.060,31$ & 466,28 \\
\hline 2016 & 2,19 & $1.217,85$ & 964,04 & 477,31 \\
\hline Variação & $-24,48$ & $-7,90 \%$ & $-9,08 \%$ & $-2,36 \%$ \\
\hline
\end{tabular}




\section{ARTIGO ORIGINAL}

\section{CAROLINA MARTINS COSTA MAIRA OLIVEIRA BITENCOURT ALEXANDRE FERREIRA BELLO ALEXANDRE PAIM DIAZ}
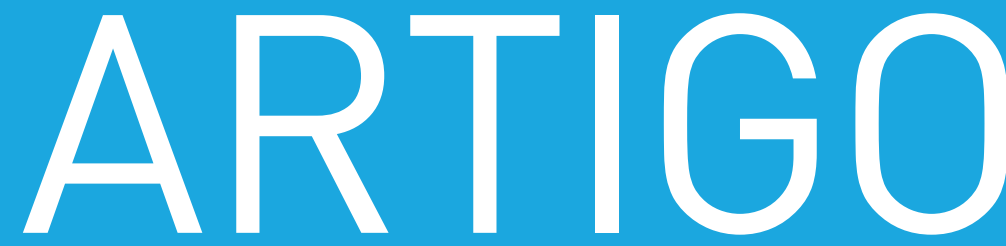

Na análise de regressão linear, houve um aumento significativo das taxas de suicídio em homens dos 60 aos 74 anos de idade e acima dos 80 anos. Para o sexo feminino, houve aumento significativo nas taxas de mortalidade por suicídio dos 60 aos 69 anos de idade. Em relação às outras causas de mortalidade, houve redução significativa nas taxas de mortalidade por doenças cerebrovascular, doença isquêmica do coração e doença crônica de vias aéreas inferiores em ambos os sexos na faixa etária entre 60 e 74 anos (Tabela 2). As Figuras 1 e

Tabela 2 - Análise de regressão linear, tendo como variável independente o ano (de 1996 a 2016) e como variável dependente a taxa de mortalidade

\begin{tabular}{|c|c|c|c|c|}
\hline \multirow[b]{2}{*}{ Faixa etária } & \multicolumn{4}{|c|}{ Causas de mortalidade } \\
\hline & $\begin{array}{c}\text { Morte por lesão } \\
\text { autoprovocada } \\
\text { Coeficiente B (IC95\%) }\end{array}$ & $\begin{array}{c}\text { Doença } \\
\text { cerebrovascular } \\
\text { Coeficiente B (IC95\%) }\end{array}$ & $\begin{array}{c}\text { Doença isquêmica } \\
\text { do coração } \\
\text { Coeficiente B (IC95\%) }\end{array}$ & $\begin{array}{c}\text { Doença crônica de } \\
\text { vias aéreas inferiores } \\
\text { Coeficiente B (IC95\%) }\end{array}$ \\
\hline \multicolumn{5}{|c|}{$\begin{array}{l}\text { Geral (a partir dos } 60 \\
\text { anos de idade) }\end{array}$} \\
\hline Total & $0,06(0,02$ a 0,11$)$ & $-5,06(-6,40$ a $-3,72)$ & $-1,84(-3,36$ a $-0,33)$ & $-1,94(-2,89$ a $-0,99)$ \\
\hline Feminino & $0,03(0,01$ a 0,05$)$ & $-4,64(-5,97$ a $-3,31)$ & $-2,56(-3,91$ a $-1,21)$ & $-0,53(-1,13$ a 0,27$)$ \\
\hline Masculino & $0,12(0,04$ a 0,21) & $-5,47(-6,84 a-4,10)$ & $-0,72(-2,42$ a 0,99$)$ & $-3,54(-4,72$ a $-2,36)$ \\
\hline \multicolumn{5}{|l|}{60 a 64 anos } \\
\hline Total & $0,07(0,02$ a 0,11) & $-3,93(-4,51$ a $-3,36)$ & $-1,61(-2,33$ a $-0,89)$ & $-1,35(-1,69$ a $-1,01)$ \\
\hline Feminino & $0,07(0,04$ a 0,10) & $-3,12(-3,50$ a $-2,64)$ & $-1,60(-2,09$ a -1,11) & $-0,64(-0,93$ a $-0,36)$ \\
\hline Masculino & $0,07(0,001$ a 0,15$)$ & $-4,83(-5,53$ a $-4,13)$ & $-1,56(-2,56$ a $-0,57)$ & $-2,13(-2,55$ a $-1,72)$ \\
\hline \multicolumn{5}{|l|}{65 a 69 anos } \\
\hline Total & $0,09(0,02$ a 0,16$)$ & $-5,42(-6,24 a-4,58)$ & $-2,03(-3,11$ a $-0,95)$ & $-2,27(-2,84 a-1,70)$ \\
\hline Feminino & $0,06(0,03$ a 0,09$)$ & $-4,55(-5,24$ a $-3,86)$ & $-2,17(-3,00$ a $-1,35)$ & $-0,93(-1,35$ a $-0,51)$ \\
\hline Masculino & $0,13(0,002$ a 0,25) & $-6,39(-7,40$ a $-5,37)$ & $-1,80(-3,22$ a $-0,37)$ & $-3,80(-4,59 a-3,01)$ \\
\hline \multicolumn{5}{|l|}{70 a 74 anos } \\
\hline Total & $0,06(0,02$ a 0,10) & $-9,15(-10,46$ a $-7,85)$ & $-5,22(-6,57$ a $-3,87)$ & $-4,16(-5,01$ a $-3,31)$ \\
\hline Feminino & - 0,006 (-0,03 a 0,02) & $-8,05(-9,20$ a $-6,90)$ & $-5,45(-6,70$ a $-4,19)$ & $-1,69(-2,32$ a $-1,05)$ \\
\hline Masculino & $0,16(0,08$ a 0,024$)$ & $-10,24(-11,72$ a $-8,76)$ & $-4,60(-6,04 a-3,15)$ & $-6,98(-8,10$ a $-5,86)$ \\
\hline \multicolumn{5}{|l|}{75 a 79 anos } \\
\hline Total & $0,03(-0,06$ a 0,11$)$ & $-11,74(-14,71$ a $-8,77)$ & $-5,50(-8,47$ a $-2,52)$ & $-4,65(-6,46 a-2,83)$ \\
\hline Feminino & $0,004(-0,03$ a 0,04$)$ & $-11,57(-14,45$ a $-8,69)$ & $-6,97(-9,63 a-4,31)$ & $-2,01(-3,48$ a $-0,55)$ \\
\hline Masculino & $0,09(-0,07$ a 0,26$)$ & $-11,40(-14,52$ a $-8,27)$ & $-2,92(-6,25$ a 0,42$)$ & $-7,51(-9,85 a-5,16)$ \\
\hline \multicolumn{5}{|c|}{ Acima de 80 anos } \\
\hline Total & $0,15(0,09$ a 0,21$)$ & $-9,44(-15,87$ a $-3,01)$ & $-3,62(-9,31$ a 2,07$)$ & $-3,82(-7,88$ a 0,23) \\
\hline Feminino & - 0,02 (-0,06 a 0,01) & $-13,01(-19,51$ a $-6,50)$ & $-8,67(-14,06$ a $-3,29)$ & $-2,22(-5,43$ a 0,99$)$ \\
\hline Masculino & $1,29(1,13$ a 1,44$)$ & $-3,68(-10,27$ a 2,91$)$ & $4,52(-1,82$ a 10,86$)$ & $-5,17(-10,72$ a 0,39$)$ \\
\hline
\end{tabular}


${ }^{1}$ Faculdade de Medicina, Universidade do Sul de Santa Catarina (UNISUL), Palhoça, SC. ${ }^{2}$ Instituto de Psiquiatria de Santa Catarina, São José, SC. ${ }^{3}$ Universidade Federal de Santa Catarina (UFSC), Florianópolis, SC.

2 mostram a tendência das taxas de mortalidade a cada 5 anos do período estudado para os sexos masculino e feminino na faixa etária entre 65 e 69 anos.

\section{Discussão}

Neste estudo, encontramos um aumento significativo nas taxas de suicídio para homens e mulheres entre 60 e 69 anos de idade em duas décadas de observação. Por outro lado, para a mesma faixa etária e ambos os sexos, houve redução significativa nas taxas de mortalidade por doença cerebrovascular, doença isquêmica do coração e doença crônica de vias aéreas superiores, três das principais causas de mortalidade em 1996, ano de início do período de observação correspondente a esta pesquisa.
Os idosos apresentam as taxas de suicídio mais elevadas em vários países ${ }^{11,12}$. Esse maior risco de suicídio tem sido associado a vários fatores, por exemplo, redução na integração social, maior prejuízo na saúde física, de mobilidade e maior prevalência de doenças terminais ${ }^{13,14}$. No Brasil, Minayo et al. analisaram as taxas de suicídio entre 1980 e 2006, apresentando evidências de maiores taxas entre os idosos do sexo masculino nesse período ${ }^{15}$. Outro estudo mostrou que entre 2000 e 2012 as taxas de suicídio aumentaram 16,2\% no Brasil, com taxas três vezes maiores para os homens em comparação às mulheres, as quais também apresentaram aumento significativo ao longo do tempo ${ }^{16}$. Rodrigues et al. analisaram a tendência das taxas de suicídio no Brasil de 1997 a $2015^{10}$. Os resultados mostraram que a população

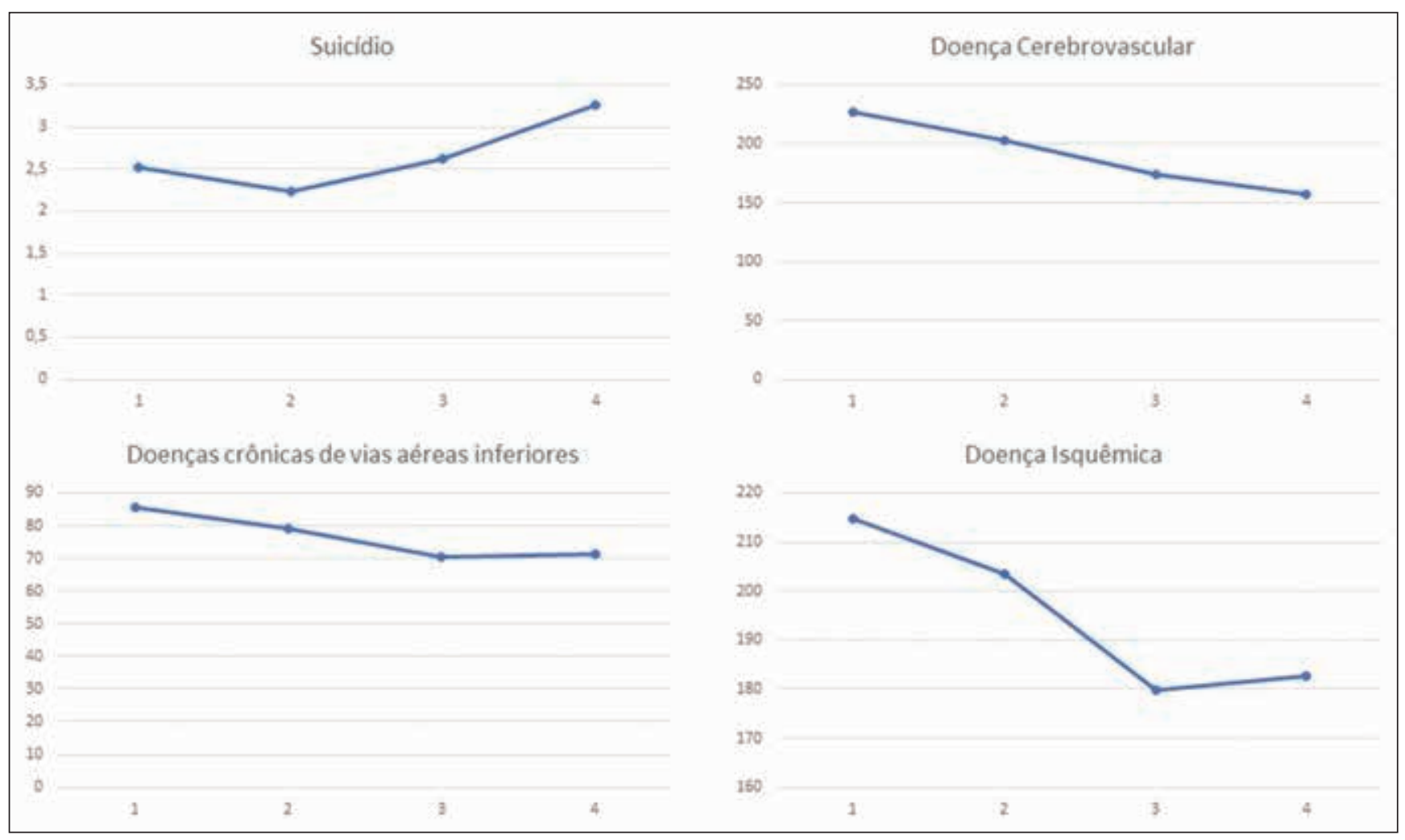

Figura 1 - Taxas de mortalidade/100.000 habitantes para o sexo feminino na faixa etária dos 65 aos 69 anos, por causa. 1 = taxa média de mortalidade/100.000 habitantes de 1996 a 2000; 2 = taxa média de mortalidade/100.000 habitantes de 2001 a 2005; 3 = taxa média de mortalidade/100.000 habitantes de 2006 a 2010; 4 = taxa média de mortalidade/100.000 habitantes de 2011 a 2016. 


\section{ARTIGO ORIGINAL}

\section{CAROLINA MARTINS COSTA MAIRA OLIVEIRA BITENCOURT ALEXANDRE FERREIRA BELLO ALEXANDRE PAIM DIAZ}

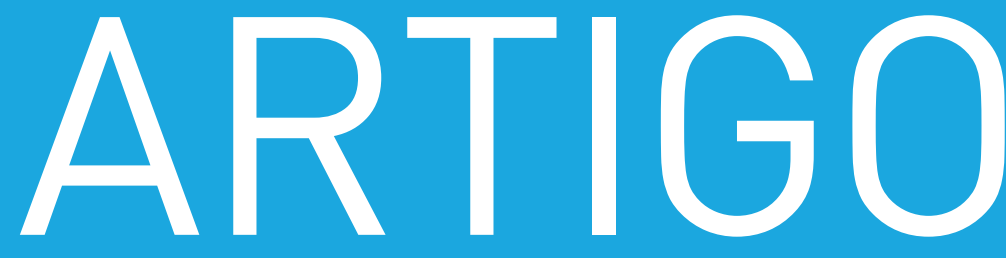

com 60 anos ou mais apresentou a maior taxa de suicídio, com uma tendência crescente no período analisado. De acordo com os autores, com o aumento observado das taxas, é possível que o Brasil não alcance os objetivos de redução da mortalidade por suicídio sugeridos pela Organização Mundial de Saúde até o ano de 202010. Mesmo a China, que tem apresentado uma redução nas suas taxas nacionais de suicídio, apresenta tendência aumentada quanto analisada apenas a população idosa ${ }^{17}$. No presente estudo, as taxas de mortalidade por suicídio em idosos aumentaram significativamente tanto em homens quanto em mulheres, principalmente na faixa etária dos 60 aos 69 anos de idade, sendo maior em homens para todas as faixas etárias analisadas.

Houve um declínio constante nas taxas de suicídio em idosos em Singapura de 1995 a 2000 ${ }^{18}$. Alguns fatores poderiam explicar a tendência de queda. Depois de 1995, houve um esforço nacional para examinar os problemas da população idosa e foi criado um comitê interministerial com representantes dos Ministérios da Saúde, do Desenvolvimento Comunitário, do Trabalho e do Desenvolvimento Nacional. Algumas questões identificadas como urgentes para as necessidades dos idosos incluíram melhoria da habitação, cuidados familiares, cuidados com a saúde, transporte e apoio comunitário. A detecção precoce e o manejo de idosos com depressão no nível da atenção primária foram enfatizados no relatório, e seminários regulares com cuidadores primários foram organizados. Profissionais de saúde que trabalhavam com idosos, médicos, enfermeiros e assistentes sociais também foram alertados sobre os idosos em risco. 0 Ministério da Saúde também começou a treinar mais

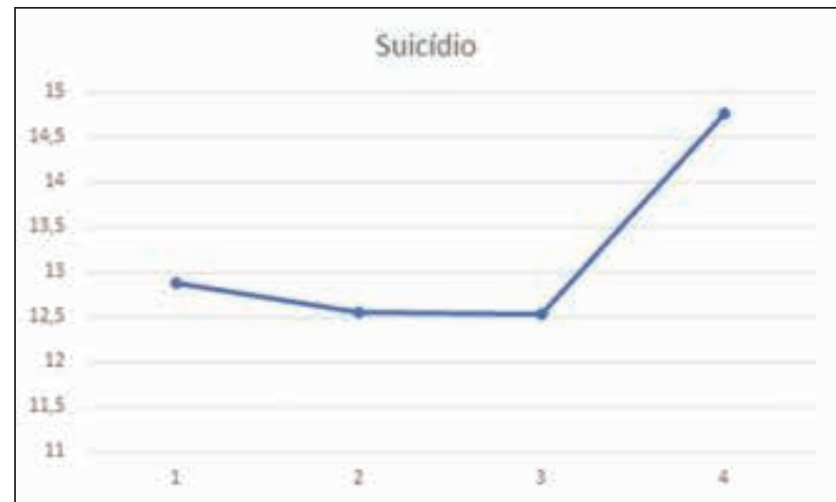

Doenças crônicas de vias aéreas inferiores
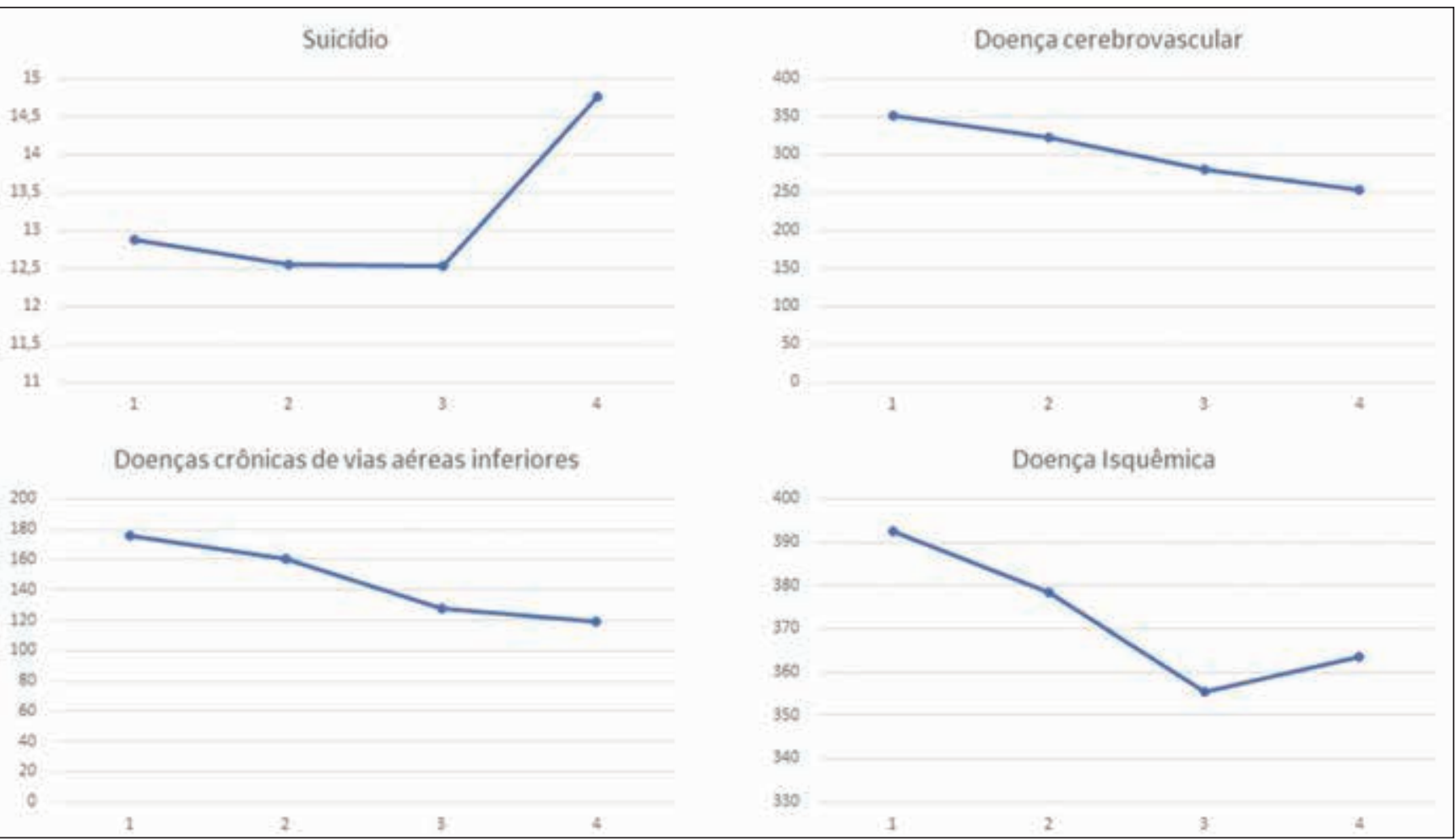

Figura 2 - Taxas de mortalidade/100.000 habitantes para o sexo masculino na faixa etária dos 65 aos 69 anos, por causa. 1 = taxa média de mortalidade/100.000 habitantes de 1996 a 2000; 2 = taxa média de mortalidade/100.000 habitantes de 2001 a 2005; 3 = taxa média de mortalidade/100.000 habitantes de 2006 a 2010; 4 = taxa média de mortalidade/100.000 habitantes de 2011 a 2016. 


\section{CAROLINA MARTINS COSTA ${ }^{1}$, MAIRA OLIVEIRA BITENCOURT ${ }^{2}$ ALEXANDRE FERREIRA BELLO ${ }^{1}$, ALEXANDRE PAIM DIAZ ${ }^{3}$}

${ }^{1}$ Faculdade de Medicina, Universidade do Sul de Santa Catarina (UNISUL), Palhoça, SC. ${ }^{2}$ Instituto de Psiquiatria de Santa Catarina, São José, SC. ${ }^{3}$ Universidade Federal de Santa Catarina (UFSC). Florianópolis, SC.

médicos e enfermeiros em medicina geriátrica e psiquiatria geriátrica, e uma linha direta de telefone foi criada por uma organização voluntária para idosos, a Singapore Action Group of Elders (SAGE) ${ }^{18}$. Singapura não é o único país a criar essa linha direta e ter bons resultados. Um estudo italiano mostrou que a introdução de um serviço de aconselhamento por telefone ajudou a reduzir as taxas de suicídio de idosos ${ }^{19}$.

No que tange às outras causas de mortalidade em idosos, as tendências diferem bastante das tendências de mortalidade por suicídio. Assim como no presente estudo, a mortalidade por acidente vascular cerebral (AVC), doenças isquêmicas e doenças pulmonares têm apresentado tendência decrescente em outros países. Em estudo realizado no Mississipi, EUA, em que foram analisadas as tendências de mortalidade por AVC na população, o declínio foi significativo entre adultos com idade acima de 65 anos $^{20}$. Pesquisadores documentaram várias razões para o declínio nas taxas de morte por acidente vascular cerebral, como o controle aprimorado de fatores de risco, como hipertensão arterial e dislipidemia ${ }^{21}$. Em outro estudo de tendência temporal de mortalidade por doenças isquêmicas do coração (DIC), entre os anos de 1980 e 2010, concluiu-se que, globalmente, a mortalidade por DIC padronizada por idade diminuiu desde os anos 80 , e as regiões de alta renda (especialmente a Austrália, a Europa Ocidental e a América do Norte) experimentaram os declínios mais notáveis ${ }^{22}$.

Na maioria das regiões do mundo, particularmente em regiões de alta renda, as taxas de mortalidade por DIC padronizadas por idade diminuíram significativamente desde $1980^{22}$. A mortalidade por DIC padronizada pela idade diminuiu em grande parte devido à combinação de melhor prevenção primária (melhorias nos fatores de risco) e melhor prevenção secundária (melhor tratamento de DIC aguda e crônica) ${ }^{23}$. Em estudo mundial, houve uma clara queda nas taxas específicas por idade e sexo para todos os grupos entre 1990 e 2010 nas taxas de mortalidade por doença pulmonar ${ }^{24}$. Muito deste declínio esteve associado a mudanças na renda nacional bruta (RNB). Em países com maior incremento na RNB, houve redução relativa nas taxas de mortalidade por doença pulmonar obstrutiva crônica (DPOC). Mudanças ao longo do tempo na exposição cumulativa ao consumo de cigarros tiveram relativamente pouco impacto. Além disso, a forte associação com pobreza, particularmente nas regiões mais carentes do mundo, demonstram que a redução da pobreza é de extrema relevância na reversão da mortalidade por DPOC 24 .

Nosso estudo tem limitações que devem ser assinaladas. Como trata-se de um estudo ecológico, os resultados devem ser interpretados com cautela, já que faltam características individuais da população estudada, como, por exemplo, a prevalência de transtornos psiquiátricos e outras variáveis associadas ao risco de suicídio e às outras causas de mortalidade. Outra limitação é em relação ao registro das certidões de óbito, com possível subnotificação de casos de suicídio. Não foram também estratificadas taxas de óbitos para regiões do país, o que, para um país continental e heterogêneo como o Brasil, poderia mostrar tendências particulares.

Concluindo, no Brasil, observamos uma tendência significativa de aumento nas taxas de suicídio em idosos entre 60 e 69 anos de idade no período de 1996 a 2016, apesar de uma redução significativa de outras importantes causas de mortalidade, como doença cerebrovascular, doença isquêmica do coração e doença de vias aéreas inferiores. É possível que políticas públicas específicas para a redução dos fatores de risco associados ao suicídio tenham o mesmo impacto que aquelas implementadas para a redução da mortalidade por outras causas. Esforços nacionais têm sido implementados nos últimos anos, tanto do ponto de vista institucional como por parte da sociedade civil organizada e associações de especialidade. Recentemente, o Ministério da Saúde do Governo Federal lançou a Agenda de Ações Estratégicas para a Vigilância e Prevenção do Suicídio e Promoção da Saúde no Brasil, com o objetivo de redução das taxas de suicídio nos próximos anos ${ }^{25}$. Além disso, a Associação Brasileira de Psiquiatria (ABP), por meio da campanha Setembro Amarelo, tem promovido diversas ações no sentido de conscientizar a sociedade sobre a importância de redução do estigma em relação aos transtornos psiquiátricos e psicoeducação sobre os principais fatores de risco, proteção e sinais de alerta para o risco de suicídio, também com o intuito de reduzir os índices de mortalidade por suicídio na população brasileira. Com essas ações, espera-se que a mortalidade por suicídio, não apenas na população idosa, mas como um todo, apresente a mesma tendência decrescente de mortalidade como aquela observada por outras causas. 
Artigo submetido em 20/10/2019, aceito em 21/10/2019. Os autores informam não haver conflitos de interesse associados à publicação deste artigo.

Fontes de financiamento inexistentes.

Correspondência: Alexandre Paim Diaz, Núcleo de Psiquiatria, Hospital Universitário da UniversidadeFederal de Santa Catarina, Campus Universitário, Rua Professora Maria Flora Pausewang, s/nº, Trindade, CEP 88036-800, Florianópolis, SC. E-mail: alexandrepaimdiaz@gmail.com

\section{Referências}

1. Veras R. Population aging today: demands, challenges and innovations. Rev Saude Publica. 2009;43:548-54.

2. Hedegaard H, Curtin SC, Warner M. Suicide mortality in the United States, 1999-2017. NCHS Data Brief. 2018;(330):1-8.

3. Minayo MC, Cavalcante FG, de Souza ER. Methodological proposal for studying suicide as a complex phenomenon. Cad Saude Publica. 2006;22:1587-96.

4. Minayo MC, Cavalcante FG. Suicide in elderly people: a literature review. Rev Saude Publica. 2010;44:750-7.

5. Otte C, Gold SM, Penninx BW, Pariante CM, Etkin A, Fava M, et al. Major depressive disorder. Nat Rev Dis Primers. 2016;2:16065.

6. Stickley A, Koyanagi A. Loneliness, common mental disorders and suicidal behavior: findings from a general population survey. J Affect Disord. 2016;197:81-7.

7. Minayo MC, Meneghel SN, Cavalcante FG. [Suicide of elderly men in Brazil]. Cien Saude Colet. 2012;17:2665-74.

8. Cuthbert BN, Insel TR. Toward the future of psychiatric diagnosis: the seven pillars of RDoC. BMC Med. 2013;11:126.

9. Carmo EA, Santos PH, Ribeiro BS, Soares CJ, Santana ML, Bomfim ED, et al. Sociodemographic characteristics and time series of mortality due to suicide among elderly individuals in Bahia State, Brazil, 1996-2013. Epidemiol Serv Saude. 2018;27:e20171971.

10. Rodrigues CD, de Souza DS, Rodrigues HM, Konstantyner TC. Trends in suicide rates in
Brazil from 1997 to 2015. Braz J Psychiatry. 2019;41:380-8.

11. Varnik P. Suicide in the world. Int J Environ Res Public Health. 2012;9:760-71.

12. World Health Organization. Preventing suicide: a global imperative [Internet]. 2014. www.who. int/mental_health/suicide-prevention/world_ report_2014/en/.

13. Phillips JA. A changing epidemiology of suicide? The influence of birth cohorts on suicide rates in the United States. Soc Sci Med. 2014;114:15160.

14. Stack S. Social correlates of suicide by age. In: Leenaars AA, editor. Time-Lines in the Suicide Process. Boston: Springer; 1991; p. 187-213.

15. Minayo MC, Pinto LW, Assis SG, Cavalcante FG, Mangas RM. Trends in suicide mortality among Brazilian adults and elderly, 1980-2006. Rev Saude Publica. 2012;46:300-9.

16. Machado DB, dos Santos DN. Suicídio no Brasil, de 2000 a 2012. J Bras Psiquiatr. 2015;64:4554.

17. Wang CW, Chan CL, Yip PS. Suicide rates in China from 2002 to 2011: an update. Soc Psychiatry Psychiatr Epidemiol. 2014:49:929-41.

18. Kua EH, Ko SM, Ng TP. Recent trends in elderly suicide rates in a multi-ethnic Asian city. Int J Geriatr Psychiatry. 2003;18:533-6.

19. De Leo D, Carollo G, Dello Buono M. Lower suicide rates associated with a Tele-Help/TeleCheck service for the elderly at home. Am J Psychiatry. 1995;152:632-4.

20. Mendy VL, Vargas R, Payton M, Sims JN, Zhang L. Trends in the stroke death rate among Mississippi adults, 2000-2016. Prev Chronic Dis. 2019;16:E21.

21. Lackland DT, Roccella EJ, Deutsch AF, Fornage M, George MG, Howard G, et al. Factors influencing the decline in stroke mortality: a statement from the American Heart Association/American Stroke Association. Stroke. 2014;45:315-53.

22. Moran AE, Forouzanfar MH, Roth GA, Mensah GA, Ezzati M, Murray CJ, et al. Temporal trends in ischemic heart disease mortality in 21 world regions, 1980 to 2010: the Global Burden of 
CAROLINA MARTINS COSTA', MAIRA OLIVEIRA BITENCOURT ${ }^{2}$,

ALEXANDRE FERREIRA BELLO ${ }^{1}$, ALEXANDRE PAIM DIAZ ${ }^{3}$

${ }^{1}$ Faculdade de Medicina, Universidade do Sul de Santa Catarina (UNISUL), Palhoça, SC. ${ }^{2}$ Instituto de Psiquiatria de Santa Catarina, São José, SC. ${ }^{3}$ Universidade Federal de Santa Catarina (UFSC), Florianópolis, SC.

Disease 2010 study. Circulation. 2014;129:148392.

23. Tunstall-Pedoe H, Vanuzzo D, Hobbs M, Mahonen M, Cepaitis Z, Kuulasmaa K, et al. Estimation of contribution of changes in coronary care to improving survival, event rates, and coronary heart disease mortality across the WHO MONICA Project populations. Lancet. 2000;355:688-700.
24. Burney PG, Patel J, Newson R, Minelli C, Naghavi M. Global and regional trends in COPD mortality, 1990-2010. Eur Respir J. 2015;45:1239-47.

25. Brasil, Ministério da Saúde. Agenda de ações estratégicas para a vigilância e prevenção do suicídio e promoção da saúde no Brasil: 2017 a 2020. 2017 [cited 2019 Nov 27]. www.neca. org.br/wp-content/uploads/cartilha_agendaestrategica-publicada.pdf 